\title{
X-Ray Magnetic Circular Dichroism of Size-Selected, Thiolated Gold Clusters
}

Yuichi Negishi, ${ }^{\dagger}$ Hironori Tsunoyama, ${ }^{\dagger}$ Motohiro Suzuki, ${ }^{\ddagger}$ Naomi Kawamura, ${ }^{\ddagger}$ Michio, M. Matsushita, ${ }^{\sharp}$ Koichi Maruyama, ${ }^{\S}$ Tadashi Sugawara, ${ }^{\#}$ Toshihiko Yokoyama ${ }^{*, \S}$ and Tatsuya Tsukuda ${ }^{*, \dagger}$

${ }^{\dagger}$ Research Center for Molecular-Scale Nanoscience, Institute for Molecular Science, Myodaiji, Okazaki 444-8585, Japan, ${ }^{\ddagger}$ Japan Synchrotron Radiation Research Institute (JASRI/Spring-8), 1-1-1 Kouto, Sayo 679-5198, Japan, ${ }^{\S}$ Department of Molecular Structures, Institute for Molecular Science, Myodaiji, Okazaki 444-8585, Japan, and ${ }^{\#}$ Department of Basic Sciences, Graduate School of Arts and Sciences, The University of Tokyo, Komaba, Meguro-ku, Tokyo 153-8902, Japan.

\section{SQUID measurements}

Magnetization and susceptibility measurements of Au:SG clusters were performed using a SQUID magnetometer (Quantum Design, MPMS-5XL) in the temperature range of 2-290 K and at a magnetic field of 0-5 T. Figure S1 shows the paramagnetic behavior of $\mathrm{Au}_{18}(\mathrm{SG})_{14}$ with an applied field of $0.5 \mathrm{~T}$ which were obtained by subtracting contributions from a diamagnetic component. On the basis of the maximum value of the curie constant $(\chi T=0.049$ emu $\mathrm{K} \mathrm{mol}^{-1}$ ), a magnetic moment per Au-S bond in $\mathrm{Au}_{18}(\mathrm{SG})_{14}$ was estimated to be $\mu_{\mathrm{Au}-\mathrm{S}}=0.0093 \mu_{\mathrm{B}}$.

(a)

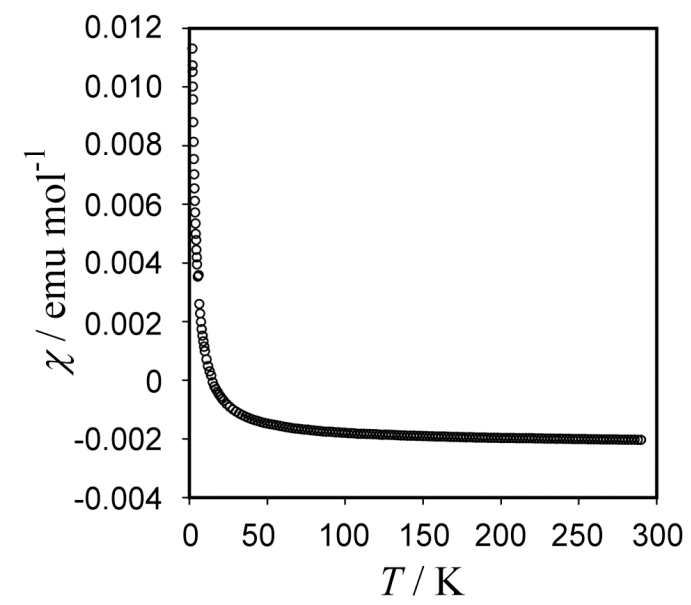

(b)

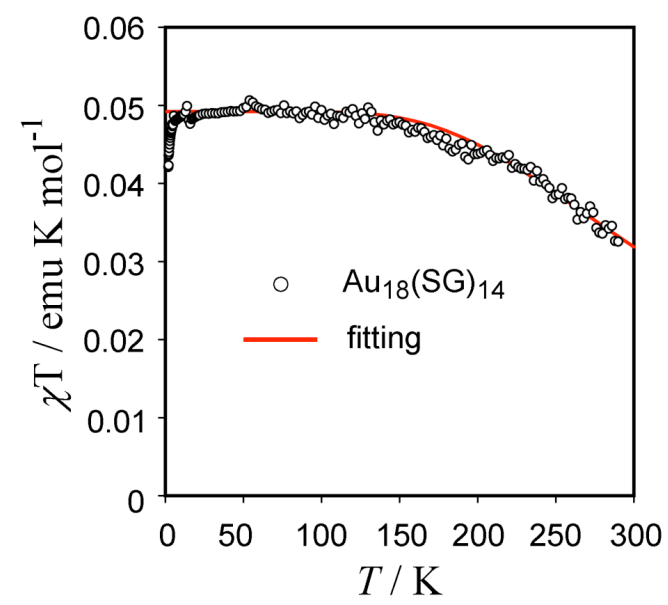

Figure S1. Temperature dependence of magnetic susceptibility of $\mathrm{Au}_{18}(\mathrm{SG})_{14}$ clusters. (a) $\chi$ vs. $T$ plot. (b) $\chi T$ vs. $T$ plot after subtracting diamagnetic component.

The sharp decrease of the $\chi \mathrm{T}$ value at lower temperatures is presumably caused by the antiferromagnetic interaction between induced electronic spins, whereas the gradual decrease in higher temperature region may be due to the thermal activation to the spinless charge-transfer excited states. The decrease of magnetic susceptibility was well reproduced using the following equation (SI-1) with values of Curie constant $(C)=0.049$, number of spinless excited states $(n)=14$, and the activation energy $\left(E_{a} / k_{B}\right)=980 \mathrm{~K}\left(8.1 \mathrm{~kJ} \mathrm{~mol}^{-1}\right)$. This result suggests that the paramagnetic spin does not originate from the impurity but is an intrinsic nature of Au:SG clusters.

$$
\chi=C \times \frac{1}{1-n \times \exp \left(-E a / k_{B} T\right)}
$$


Figure $\mathrm{S} 2$ shows the magnetization curves of $\mathrm{Au}_{18}(\mathrm{SG})_{14}$ at 2, 5, 200, $290 \mathrm{~K}$ which were obtained by subtracting contributions from a diamagnetic component. It does not exhibit hysteretic behavior and suggests the paramagnetic nature of the $\mathrm{Au}: \mathrm{SG}$ clusters.

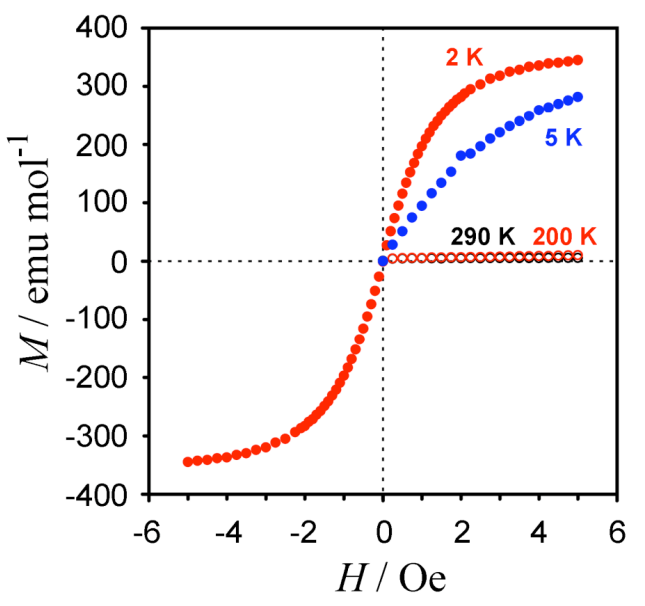

Figure S2. Magnetization curves of $\mathrm{Au}_{18}(\mathrm{SG})_{14}$ clusters at 2, 5, 200, and 290 K. 